\title{
Differential Effects of Bisphenol A and Di (2-Ethylhexyl) Phthalate Exposure on Crestin and the Expression of Some Genes Related to Apoptosis and Inflammation in Zebrafish Embryos
}

\author{
Perihan Seda Ates-Kalkan' (D), Tugce Ayik² (D), Unsal Veli Ustundag³ (D), \\ Ebru Emekli-Alturfan² (i) \\ 'University of Oxford, Radcliffe Department of Medicine, Oxford, England \\ ${ }^{2}$ Marmara University, Faculty of Dentistry, Deparment of Basic Medical Sciences, Istanbul, Turkey \\ ${ }^{3}$ Istanbul Medipol University, Faculty of Medicine, Department of Medical Biochemistry, Istanbul, Turkey
}

ORCID IDs of the authors: P.S.A.K. 0000-0002-4905-1912; T.A. 0000-0001-9655-2122; U.V.U. 0000-0003-0804-1475; E.E.A. 0000-0003-2419-8587

Please cite this article as: Ates-Kalkan PS, Ayik T, Ustundag UV, Emekli-Alturfan E. Differential Effects of Bisphenol A and Di (2-Ethylhexyl) Phthalate Exposure on Crestin and the Expression of Some Genes Related to Apoptosis and Inflammation in Zebrafish Embryos. Eur J Biol 2021; 80(1): 42-47. DOI: 10.26650/EurJBiol.2021.912385

\section{ABSTRACT}

Objective: Endocrine disrupting chemicals (EDC) in plastics may disrupt proper endocrine system functioning. Zebrafish embryos are formed through external fertilization, and their rapid development, short life cycle, and transparency provide imaging advantages. In zebrafish embryos, neural crest development occurs similarly to other vertebrate embryos and crestin is found in the neural crest during embryogenesis. The aim of our study is to evaluate the effects of bisphenol A (BPA) and $\mathrm{Di}$ (2-ethylhexyl) phthalate (DEHP), which are the most widely used EDCs, on the expression of crestin, apoptosis, and inflammation-related parameters in zebrafish embryos.

Materials and Methods: The embryos were exposed to either DEHP or BPA in well plates for $72 \mathrm{~h}$ post fertilization (hpf). Expressions of crestin were evaluated by in situ hybridization, while the expressions of bax, casp8, casp3a, ifng1, fas, and tp53 were evaluated by RT-PCR.

Results: Expressions of bax and casp 8 increased and casp $3 a$, ifng1, and fas decreased in BPA and DEHP groups. tp 53 expression increased in the BPA group but decreased in the DEHP group compared with the control group. In the DEHP group, casp3a, ifng1, fas, bax, casp8, and tp53 expressions decreased compared with the BPA group. No significant change was observed in the crestin expressions in the groups. When compared with the control group, an inverse relation between ifng1 expression and apoptosis, as evidenced by increased bax and casp8 expressions, was observed in the BPA and DEHP groups.

Conclusion: Our study provided important data on the effects of EDCs on the relationship between inflammation and apoptosis.

Keywords: Bisphenol A, Di (2-ethylhexyl) phthalate, Apoptosis, Inflammation, Crestin

\section{INTRODUCTION}

Containers and packages made of plastic are widely used in the food industry because they are lighter, unbreakable and more affordable. However, the possibility of transferring toxic additives to foods and beverages through the monomers contained in plastics has become a serious cause of concern and risk in terms of public health. Factories producing plastic and plastic derivatives release various chemicals into nature in an uncontrolled manner, and discussions about the possible effects of these chemicals on living things are in- 
creasing day by day (1). Chemicals commonly found in plastics and derivatives that disrupt endocrine functions are called endocrine disrupting chemicals (EDC) (2). In this study we aimed to examine apoptosis and inflammation-related parameters in zebrafish embryos exposed to bisphenol A (BPA) and Di (2-ethylhexyl) phthalate (DEHP), which are the most widely used EDCs.

Polyethylene terephthalate (PET) is widely used in the production of plastic materials and bottles. DEHP is used as a plasticizing agent in PET bottles. Polycarbonate plastic (PC) is widely used, especially in food and medical equipment, and BPA is needed for its production $(1,2)$. EDC exposure, which has become one of the important public health problems of our time, is related to various diseases, such as cancer. On the other hand, metabolic disease such as obesity and diabetes have also been connected to EDC exposure. It has been shown that BPA can pass into foods when it comes into contact, and this transition increases with heat. It has been shown that BPA consumed through plastic bottles can cause serious damage to the prostate, liver, and reproductive system and brain development and may lead to cancer and heart diseases (2-5).

Zebrafish, the popular model organism of recent years, is a vertebrate organism. Females lay 50 to 300 eggs per day, embryos are formed with external fertilization and have rapid development, they have short life cycles, and their transparency provides imaging advantages $(6,7)$.

It has been shown in animal models that the expression of the tp53 tumor suppressor gene can be altered by the effects of various EDCs (8). It is known that steroids and sex hormones have major roles in modulating inflammatory and immune responses, so endocrine disruptors may also affect the formation of immune responses (9). In a study conducted in rats, it was shown that IFN-expression was affected by endocrine disruptors $(10,11)$. Caspase- 3 is a caspase protein encoded by the casp3 gene that interacts with caspase-8. The interaction of the fas receptor with its ligand causes the formation of the death-inducing signaling complex. Sequential caspase activation regulates the execution phase of apoptosis and $\mathrm{Bcl}-2$ inhibits Bax-triggered apoptosis (911). In this study we aimed to determine the possible effects of DEHP and BPA on tp53, ifn- $\gamma$, casp3, casp8, bcl2, bax, and fas associated with apoptosis and inflammation in zebrafish embryos.

Crestin is expressed in zebrafish during embryogenesis and it is first observed at the beginning of somitogenesis in neural crest cells of the ectoderm (12). Although BPA and DEHP have been previously demonstrated to cause several malformations in zebrafish embryos (13), the effects of BPA and DEHP on crestin, which is an important developmental marker, have not been previously reported. Accordingly, we also aimed to evaluate the effects of BPA and DEHP on crestin expression in developing zebrafish embryos.

\section{MATERIALS AND METHODS}

\section{Embryo Exposures}

Embryos were obtained from zebrafish (wild type $A B / A B$ strain) that were housed in apparently disease-free conditions. Animal husbandry and spawning were performed according to the protocols approved by the University of Marmara Institutional Animal Care and Use Committee. As the zebrafish embryos used were no more than 5 days old, no ethical approval was required for the protocols applied as stated by the Council of Europe (1986), Directive 86/609/EEC. Reverse osmosis water that contains $0.018 \mathrm{mg} \mathrm{L-1}$ Instant Ocean ${ }^{\mathrm{TM}}$ salt was used for all of the experiments. After natural spawning, fertilized embryos were gathered and staged according to their development and morphology as described previously. Zebrafish embryos were exposed to BPA and DEHP at doses below the LC50 values as determined and reported previously by Üstündağ et al. (13). Accordingly, embryos were exposed to BPA $1 \mu \mathrm{g} / \mathrm{L}$ and DEHP $2,5 \mu \mathrm{g} / \mathrm{L}$. The embryos were exposed to either DEHP or BPA in well plates for $72 \mathrm{~h}$ post fertilization ( $\mathrm{hpf}$ ). DMSO and methanol were used as the solvent control, and the embryo medium was used as the blank control. Three replicates, each containing 30 embryos, were prepared. BPA (CAS no: 80-05-7), DEHP (CAS no: 117-81-7), dimethyl sulfoxide (DMSO) (CAS no: 67-68-5), and methanol (CAS no: 67-56-1) were purchased from Sigma-Aldrich Co. (St. Louis, Missouri, USA).

\section{Gene Expression Analyses}

Embryos in each pool were placed in a $1.5 \mathrm{ml}$ microfuge tube, water was removed, and TRIzol reagent was added. Then the embryos were homogenized with a pellet pestle. RNA isolations from the embryo homogenates were made by using the RNeasy Mini kit using the Qiacube (Qiagen) instrument. cDNA synthesis from the obtained RNA samples was carried out using One Script (ABM). The expression of bax (forward primer, 5'-GGCTATTTCAACCAGGGTTCC-3'; reverse primer, 5'-TGCGAATCACCAATGCTGT-3') casp3 (forward primer, 5'-ATGAACGGAGACTGTGTG-3'; reverse primer, 5'-TTAAGGAGTGAAGTACATCTCTTTG-3') casp8 (forward primer, 5'-GCTTCAATGGGTGCTTTTGT-3'; reverse primer, 5'-TCCGGCAAAAGGCAGTGTAA-3'), ifng1 (forward primer, 5'-GCTGGATCTTCAAAGTCGGGTGTA-3'; reverse primer, 5'-TGTGAGTCTCAGCACACTTCCATC-3'), fas (forward primer, 5'-GTGACGCTAATGCAAAAATGAAG-3'; reverse primer, 5'-CGATGTCCTGCAGAGTGGTG-3'), and tp53 (forward primer, 5'-GGGCAATCAGCGAGCAAA-3'; reverse primer 5'-GGGCAATCAGCGAGCAAA- $3^{\prime}$ ) was determined using the Rotor-Gene Q instrument by adding the obtained cDNA samples to the RT2 SYBR Green Master Mix mixture. actb1 (forward primer, 5'-AAGCAGGAGTACGATGAGTCTG-3'; reverse primer, 5'- GGTAAACGCTTCTGGAATGAC-3') was used as the housekeeping gene. The samples were studied in triplicate. Analysis of the data was performed using the delta delta Ct method based on normalization with the housekeeping gene actb1 (14).

\section{Crestin Expression Analysis by In Situ Hybridization Method} In order to determine the expression of the crestin by the in situ hybridization method, a previously prepared RNA probe targeting the crestin was used. 10 embryos reaching 72 hours were homogenized and RNA was isolated with the RNeasy mini kit. After the RNA was converted to CDNA, the desired region was amplified by PCR with previously designed crystalline primers. In order to check the accuracy of the proliferating area, it was 
validated by the gel electrophoresis method. Then, after the desired region was reproduced by PCR, unwanted molecules (dntps etc.) were removed, except for the area replicated with the clean-up kit. Then, the PCR product obtained by the in vitro transcription method was transformed into a DIG-labeled RNA. The obtained RNA was made usable by cleaning with the RNA clean-up kit. The probe obtained in this way was compared with the embryos to which BPA and DEHP had been administered by using the in situ hybridization method and the control group embryos (15).

\section{Statistical Analysis}

Statistical analysis of the data was done with the Graph Pad 7 Statistical program using Tukey Multiple Comparison Analysis after One Way Anova. The Shapiro-Wilk test was performed to test the normality. Results are given as Mean \pm Standard Deviation; $p<0.05$ was considered significant.

\section{RESULTS}

As the data analysis did not show any difference between the solvent control and blank control for the parameters investigated in this study, accordingly, the blank control test data are given in the following sections. It was determined that there was a significant increase in bax and casp8 mRNA expression levels in BPA and DEHP groups compared to the control group $(p<0.05)$. When the DEHP group was compared with the BPA group, it was observed that there was a significant decrease in bax and casp8 mRNA expression levels ( $p<0.05)$ (Figure 1).
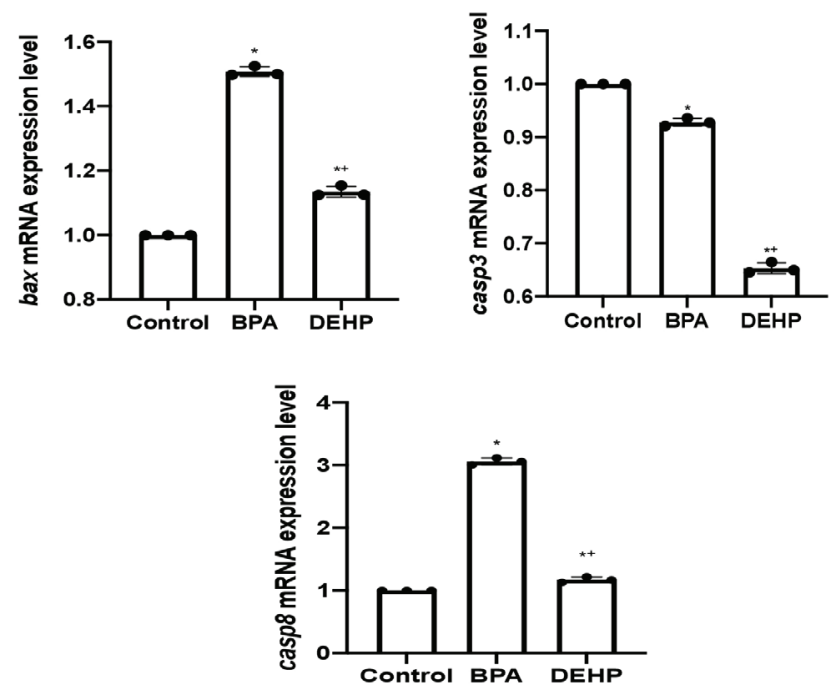

Figure 1. bax, casp3 and casp8 transcripts quantified by RT-PCR. All RT-PCR results are normalized to $\beta$-actin, the housekeeping gene and expressed as fold change from their respective controls. Data presented are mean \pm SD. *significantly different from the control group, $p<0.05$; ${ }^{+}$significantly different from the BPA group; SD: standard deviation; BPA: Bisphenol A; DEHP: Di (2-ethylhexyl) phthalate.
A significant decrease was found in casp3a, ifng1, and fas mRNA expression levels in both the BPA and DEHP groups $(p<0.05)$. In the DEHP group, a significant decrease was observed in casp3a, ifng1, and fas mRNA expression levels compared to the BPA group and compared to the control group (Figures 1 and 2) $(p<0.05)$.
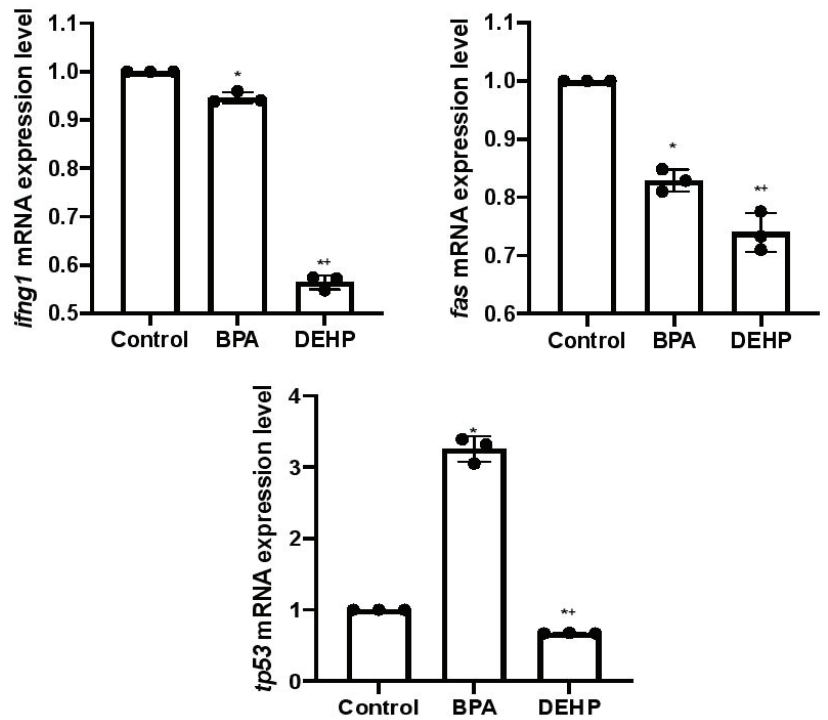

Figure 2. ifng1, fas and tp53 transcripts quantified by RT-PCR. All RT-PCR results are normalized to $\beta$-actin, the housekeeping gene and expressed as fold change from their respective controls. Data presented are mean \pm SD. *significantly different from the control group, $\mathrm{p}<0.05 ;{ }^{+}$significantly different from the BPA group; SD: standard deviation; BPA: Bisphenol A; DEHP: Di (2-ethylhexyl) phthalate.

tp53 mRNA expression levels were found to be increased in the BPA group compared to the control group $(p<0.05)$. On the other hand, in the DEHP group, a significant decrease was observed in the tp53 mRNA expression level compared to both the control and BPA groups $(\mathrm{p}<0.05)$ (Figure 2$)$. Crestin-expressing cells were found to be distributed along the anterior-posterior axis, in the neural crest migratory pathways. No significant change was observed in the crestin expressions in the groups (Figure 3).

\section{DISCUSSION}

In our study, we examined the effects of exposure to BPA and DEHP on the expression of apoptosis-associated p53, casp3, casp8, and bax genes and the inflammation-related ifn- $\gamma$ gene, as well as the expression of crestin, which is an important marker in the embryonic period.

BPA is used to produce polycarbonate plastic and it acts as synthetic estrogen. BPA is found in different products such as food containers, toys, baby bottles, and dental and medical devices. Plastics are widely used in modern life and therefore 


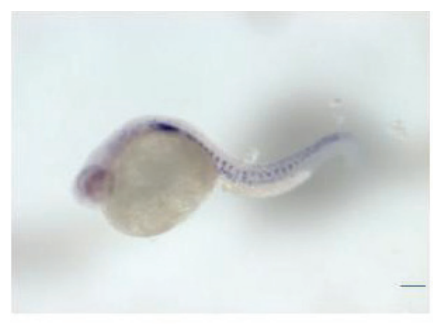

Control

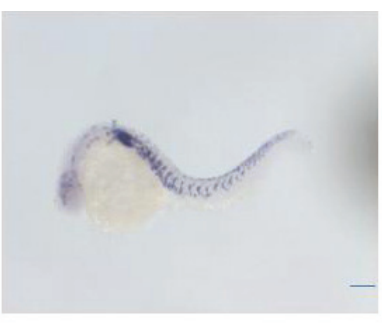

BPA

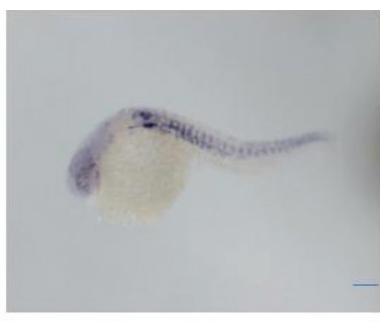

DEHP

Figure 3. Crestin expressions of the control, Bisphenol A (BPA) and Di (2-ethylhexyl) phthalate (DEHP) groups determined by in situ hybridization. Crestin expressing cells are distributed along the anterior-posterior axis, in the neural crest migratory pathways. Scale bar: $60 \mu \mathrm{M}$.

BPA is released into the environment directly or under normal conditions due to incomplete polymerization. Hydrolysis of the ester bonds binding BPA monomers due to acid, base, or heat exposures lead to the release of BPA and increase the migration rate. BPA can be found in water sources such as streams and rivers, and even in drinking water. The main source of exposure is suggested to be by food and drink (16). We have shown previously that BPA and DEHP induced axial malformations, pericardial edema, and yolk sac edema in the zebrafish embryos (13). BPA affects different physiological processes related to development, reproduction, and metabolism. These effects can be attributed to its estrogen-like effect, whereby it binds to the estrogen receptors, altering the hormone system. BPA exposure has been related to many diseases affected by changes in estrogen levels, such as ovarian disease and breast cancer. Moreover, body fat distribution has been shown to correlate with blood BPA levels in women (17).

Increased bax and casp8 expressions in the BPA- and DEHP-exposed groups is consistent with some other studies showing the apoptotic effects of these EDCs. Apoptosis is a programmed event that plays an important role in the normal development of living things, in the elimination of defective germ cells, in maintaining the proportion of Sertoli cells with appropriate germ cells, and in controlling sperm production. It has been reported that BPA induces autophagy and apoptosis in SertoIi and Leyding cells in rats (18). In testicular Leydig cells, it has been shown that BPA can activate protein kinase A (PKA) and Akt. In different models, BPA has been shown to affect proliferation of cells, apoptosis, and steroidogenesis by binding specific receptors in signaling pathways. On the other hand, information on the mechanism of germ cell apoptosis activated by BPA and the role of apoptosis-signaling pathways is scarce (16-18). In our study, although bax and casp8 expressions were found to be increased in the BPA and DEHP groups, casp3 expressions were found to be decreased when compared with the control group. The inhibition of casp3 may be related to developmental defects, as reported by Oka et al. (19), who aimed to evaluate how BPA affected the early development of Xenopus laevis embryos. For this purpose, they exposed the embryos to 10-100 $\mu \mathrm{M}$ BPA and reported that the developmental abnormalities were found in embryos exposed to $20 \mu \mathrm{M}$ BPA. As apoptosis is induced through various stimuli, inside the cell the apoptotic signal within the cell is transmitted through two main molecular pathways. These pathways are the death receptor pathway, known as the extrinsic-external pathway, and the mitochondrial pathway, known as the intrinsic or internal pathway. The intrinsic pathway can be induced by various extracellular or intracellular signals. These signals may include radiation, oxidative stress, and reactive oxygen species, as well as the intermediates of xenobiotic metabolism. BPA and some chemotherapeutic drugs may also lead to the dysfunction of mitochondria. Mitochondria is the control point of the intrinsic apoptotic pathway that is modulated by $\mathrm{Bcl}-2$ family proteins and results in cell death by cytochrome $c$ and other proapoptotic effectors (18).

In our study, p53 expression levels increased significantly in embryos exposed to BPA compared to the control group. Lloyd et al. (20) showed that BPA led to increased P53 protein levels through the western blot analysis method in the breast cancer cell line. Although cytolocalization of P53 was not affected by BPA administration, an increase in cell proliferation was observed. Jiang et al. (21) reported that BPA exposure increased the levels of casp 3, 8, 9, and 10 in the kidney and showed an apoptotic effect in rats with BPA added to their feed for five weeks. Xu et al. (22) studied the effect of BPA on ovarian granulosa cells in rats. They exposed ovarian granulosa cells to BPA for 24 hours to 72 hours and reported that BPA reduced the viability of granulosa cells in a dose- and time-dependent manner. Researchers have reported that BPA increases Bax expression and decreases $\mathrm{BCl} 2$ expression in granulosa cells. These results show that low doses of BPA reduce the granulosa cell viability through the induction of apoptosis. It has been suggested that increased Bax expression and decreased $\mathrm{Bcl} 2$ are effectively involved in this apoptotic effect.

The mechanism of BPA-induced apoptosis may proceed through three distinct pathways: the DNA damage response signaling pathway, the mitogen-activated protein kinase (MAPK) signaling cascade, and the insulin-like growth factor-1 signaling pathway. In our previous study, we showed that BPA increased nitric oxide levels and decreased glutathione S-transferase and superoxide dismutase activities (5). Accordingly, increased oxidative stress may be the reason for increased bax and casp8 expressions in the BPA group. 
In our study, ifng1 expression levels decreased significantly in BPA group embryos compared to the control group. Although structurally different from estrogen, BPA binds to estrogen receptor (ER) subtypes, especially ER-beta, with 6 times greater affinity. Immune system cells respond to sex hormones, including estrogen. Upon ER binding of estrogen, the ligand-receptor complex mediates the transcription activity of genes containing estrogen response elements (EREs). Estrogen regulates IFN- $\gamma$ as well as the interleukin-10 (IL-10). The ifn-promoter contains sequences similar to ERE and estrogen has been reported to activate the production of IFN- $\gamma$ (23). BPA has been shown to decrease IFN- $\gamma$ levels in both male and female mice (24).

Üstündağ et al. (13) observed increased vitellogenin levels and $w n t 3 a$ and $g s k 3$ expressions in zebrafish embryos exposed to BPA. They reported that increased apoptosis in embryos exposed to BPA can be because of Wnt3a-induced pro-apoptotic changes. However, in DEHP exposure, it has been suggested that Wnt3a has a lesser effect. In the same study, vitellogenin levels and apoptosis did not significantly increase in embryos exposed to DEHP. In the study by Üstündağ et al. (13), apoptosis was evaluated using the acridine orange method.

DEHP is toxic to zebrafish embryos and larvae and has been shown to cause developmental anomalies. It has been shown that DEHP exposure may lead to increased activity of estrogen under $1.50 \mathrm{ppm}$ and that DEHP leads to ER transactivation (25). It has been shown in previous studies that DEHP can induce apoptosis and mitochondrial damage, break the DNA strand, and lead to reactive oxygen species (ROS) production. The damage to the DNA causes the weakening of the mitochondrial function regulator known as SIRT1, and thus suppressed ATP levels. ATP is needed for spermatozoa motility, therefore decreased ATP levels caused by DEHP have been accepted as a very important problem for male fertility (26). It has been reported that the toxic effects of DEHP on ovaries and follicles may occur due to apoptosis. For proper steroidogenesis regulation and to survive atresia, follicles should function properly. Atresia is suggested to be a naturally occurring apoptotic event that occurs with the death of follicles, which detrimentally affects the reproductive system. Atresia of ovarian follicles is regulated through proapoptotic factors, including Bad and Bax, and antiapoptotic factors, including $\mathrm{BCl} 2$, which are usually inhibited due to DEHP exposure. Increased oxidative stress with DEHP may lead to follicular atresia. DEHP may lead to the inhibition of follicle growth through oxidative stress (27). In accordance with these reports in our study, we found that as a result of DEHP exposure, bax and casp8 expressions increased in zebrafish embryos compared to the control group, and casp3a, ifng 1, fas, and tp53 expressions decreased. In addition, the expressions of bax, casp3a, casp8, fas, ifng 1, and tp53 decreased when embryos exposed to DEHP were compared with those exposed to BPA.

Cell death plays an important role in the regulation of inflammation and may also be observed as a result of inflammation. For the maintenance of tissue homeostasis, both the recognition and removal of invading pathogens, as well as the clearance of dying cells, are necessary (28). Although induction of apoptosis was not initially considered an action of IFNs, in vitro studies have shown that IFN- $\alpha$, IFN- $\beta$ or IFN- $\gamma$ have induced characteristic apoptotic cell changes in the morphology of cells, such as rounding up and detachment of cells (29-31). In our study, increased bax and casp 8 expressions in the BPA and DEHP groups might be induced by IFN- $\gamma$, which in turn decreased after the induction of apoptosis. Activation of the caspase 8 cascade may lead to the release of cytochrome $\mathrm{c}$ from mitochondria, disrupt the mitochondrial potential, cause changes in the plasma membrane symmetry, and finally lead to DNA fragmentation (29-31).

In our study BPA and DEHP exposures did not change the crestin expressions. Neural crest cells develop in the third week of embryo development in humans. These cells are pluripotent in character and migrate to different places in the embryo to differentiate into different cell types. The migration of these cells is required for morphogenesis for the roles where they will function $(32,33)$. The development of the head and face region occurs as a result of more than one biological process, including the growth, proliferation, migration, and differentiation of neural crest cells $(32,33)$. The development of the neural crest occurs similarly to other vertebrate embryos in zebrafish embryos. However, since zebrafish embryos are transparent initially and have bigger but fewer neural crest cells than other species, in living embryos viable premature neural crest cells can be observed and manipulated (33). During embryogenesis, it is known that crestin is expressed in zebrafish. Crestin expression was first determined at the beginning of somitogenesis in neural crest cells of the ectoderm (33).

\section{CONCLUSION}

We have shown that as a result of BPA and DEHP exposure, while casp3a, ifng 1, and fas expressions decreased, bax and casp8 expressions increased in zebrafish embryos compared to the control group. tp53 expression increased in BPA compared to the control group, but it decreased in the DEHP group. The expressions of bax, casp3a, casp8, fas, ifng 1, and tp53 decreased when embryos exposed to DEHP were compared with those exposed to BPA. An inverse relation between ifng 1 expression and apoptosis was observed by increased bax and casp8 expressions in the BPA and DEHP groups. Accordingly, our study provided important data on the effects of EDCs on the relationship between inflammation and apoptosis.

BPA and DEHP exposures did not lead to a significant change in the crestin expressions of the embryos. However, this subject is open to research, as it is possible that different doses and different exposure durations may cause different results.

Peer-review: Externally peer-reviewed.

Conflict of Interest: The author declares that she has no conflicts of interest to disclose.

Financial Disclosure: There are no funders to report for this submission. 
Author Contributions: Conception/Design of study: E.E.A.; Data Acquisition: P.S.A.K., T.A., U.V.U.; Data Analysis/Interpretation: E.E.A.; Drafting Manuscript: E.E.A.; Critical Revision of Manuscript: E.E.A.; Final Approval and Accountability: E.E.A.

\section{REFERENCES}

1. Alabi OA, Ologbonjaye KI, Awosolu O, Alalade OE. Public and Environmental Health Effects of Plastic Wastes Disposal: A Review. J Toxicol Risk Assess 2019; 5: 021.

2. Sax L. Polyethylene terephthalate may yield endocrine disruptors. Environ Health Persp 2010; 118(4): 445-8.

3. Durusoy R, Karababa AO. Plastik gıda ambalajları ve sağlık. TAF Prevent Med Bull 2011; 10(1): 87-96.

4. Strakovsky RS, Schantz SL. Impacts of bisphenol A (BPA) and phthalate exposures on epigenetic outcomes in the human placenta. Environ Epigenet 2018; 4(3): 022.

5. Ustundag U, Unal I , Ates P, Alturfan A, Yigitbasi T, Emekli-Alturfan E. Oxidant-Antioxidant Status and c-myc Expression in BPA- and DEHP-Exposed Zebrafish Embryos. Eur J Biol 2017; 76 (1): 26-30.

6. Mudbhary R, Sadler KC. Epigenetics, development, and cancer: zebrafish make their mark. Birth Defects Res C Embryo Today 2011; 93(2): 194-03.

7. Westerfield M. The Zebrafish Book, A Guide for the Laboratory Use of Zebrafish. University of Oregon Press, Oregon, USA (1995).

8. Lee YM, Rhee JS, Hwang DS, Kim IC, Raisuddin S, Lee JS. p53 gene expression is modulated by endocrine disrupting chemicals in the hermaphroditic fish, Kryptolebias marmoratus. Comp Biochem Physiol C Toxicol Pharmacol 2008; 147(2): 150-7.

9. Diamanti-Kandarakis E, Bourguignon JP, Giudice LC, Hauser R, Prins GS, Soto AM, et al. Endocrine-disrupting chemicals: An Endocrine Society scientific statement. Endocr Rev 2009; 30(4): 293-42.

10. Jocsak G, loja E, Kiss DS, Toth I, Barany Z, Bartha T, et al. Endocrine Disruptors Induced Distinct Expression of Thyroid and Estrogen Receptors in Rat versus Mouse Primary Cerebellar Cell Cultures. Brain Sci 2019; 5;9(12): 359.

11. Anway MD, Skinner MK. Transgenerational effects of the endocrine disruptor vinclozolin on the prostate transcriptome and adult onset disease. Prostate 2008; 68(5): 517-29.

12. Mayor R, Theveneau E. The neural crest. Development 2013; 140: 2247-51.

13. Üstündağ ÜV, Ünal İ, Ateş PS, Alturfan AA, Yiğitbaşı T, Emekli-Alturfan E. Bisphenol A and di(2-ethylhexyl) phthalate exert divergent effects on apoptosis and the Wnt/ $\beta$-catenin pathway in zebrafish embryos: A possible mechanism of endocrine disrupting chemical action.Toxicol Ind Health 2017; 33(12): 901-10.

14. Livak KJ, Schmittgen TD. Analysis of relative gene expression data using real-time quantitative PCR and the 2(-Delta Delta $C(T)$ ) Method. Methods 2001; 25(4): 402-8.

15. Thisse $C$, Thisse $B$. High resolution in situ hybridization on wholemount zebrafish embryo. Nat Protoc 2008; 3: 59-69.

16. Lakind JS, Naiman DQ. Daily intake of bisphenol A and potential sources of exposure: 2005-2006 National Health and Nutrition Examination Survey. J Expo Sci Environ Epidemiol 2011; 21: 272-9.

17. Valentino R, D'Esposito V, Passaretti F, Liotti A, Cabaro S, Longo M, et al. Bisphenol-A impairs insulin action and up-regulates inflammatory pathways in human subcutaneous adipocytes and 3T3-L1 cells. PLoS ONE 2013; 8: e82099.
18. Shamas-Din A, Kale J, Leber B, Andrews DW. Mechanisms of action of Bcl-2 family proteins. Cold Spring Harb Perspect Biol 2013; 5(4): a008714.

19. Oka T, Adati N, Shinkai T, Sakuma K, Nishimura T, Kurose K. Bisphenol $A$ induces apoptosis in central neural cells during early development of Xenopus laevis. Biochem Biophys Res Commun 2003; 312(4): 877-82.

20. Lloyd V, Morse M, Purakal B, Parker J, Benard P, Crone M, et al. Hormone-like effects of bisphenol $A$ on $p 53$ and estrogen receptor alpha in breast cancer cells, BioResearch Open Access 2019; 8(1): 169-84.

21. Jiang W, Zhao H, Zhang L, Wu B, Zha Z. Maintenance of mitochondrial function by astaxanthin protects against bisphenol A-induced kidney toxicity in rats. Biomed Pharmacother 2020; 121 : 109629.

22. Xu J, Osuga $Y$, Yano T, Morita $Y$, Tang $X$, Fujiwara T, et al. Bisphenol A induces apoptosis and G2-to-M arrest of ovarian granulosa cells. Biochem Biophys Res Commun 2002; 292(2): 456-62.

23. Karpuzoglu-Sahin E, Zhi-Jun Y, Lengi A, Sriranganathan N, Ansar Ahmed $\mathrm{S}$. Effects of long-term estrogen treat- ment on IFN-gamma, IL-2 and IL-4 gene expression and protein synthesis in spleen and thymus of normal C57BL/6 mice. Cytokine 2001; 14: 208-17.

24. Sawai C, Anderson K, Walser-Kuntz D. Effect of Bisphenol A on murine immune function: Modulation of interferon- $\gamma, \operatorname{lgG} 2 \mathrm{a}$, and disease symptoms in NZB $\times$ NZW F1 mice. Environ Health Perspect 2003; 111(16).

25. Chen X, Xu S, Tan T, Lee ST, Cheng SH, Lee FW, et al. Toxicity and estrogenic endocrine disrupting activity of phthalates and their mixtures. Int JEnv Res Pub He 2014; 11(3): 3156-68.

26. Li X, Fang EF, Scheibye-Knudsen M, Cui H, Qiu L, Li J, et al. Di-(2-ethylhexyl) phthalate inhibits DNA replication leading to hyperPARylation, SIRT1 attenuation, and mitochondrial dysfunction in the testis. Sci Rep 2014; 4: 6434.

27. Wang W, Craig ZR, Basavarajappa MS, Gupta RK, Flaws JA. Di (2-ethylhexyl) phthalate inhibits growth of mouse ovarian antral follicles through an oxidative stress pathway. Toxicol Appl Pharmacol 2012; 258(2): 288-95.

28. Yang Y, Jiang G, Zhang P, Fan J. Programmed cell death and its role in inflammation. Mil Med Res 2015; $2: 12$.

29. Chawla-Sarkar M, Leaman DW, Borden EC. Preferential induction of apoptosis by interferon (IFN)- $\beta$ compared with IFN- $\alpha 2$ : Correlation with TRAIL/Apo2L induction in melanoma cell lines. Clin Cancer Res 2001; 7: 1821-31.

30. Morrison BH, Bauer JA, Kalvakolanu DV, Lindner DJ. Inositol hexakisphosphate kinase 2 mediates growth suppressive and apoptotic effects of interferon-beta in ovarian carcinoma cells. J Biol Chem 2001; 276: 24965-70.

31. Chen Q, Gong B, Mahmoud-Ahmed AS, Zhou A, Hsi ED, Hussein $M$, et al. Apo2L/TRAIL and Bcl2 related proteins regulate type I interferon induced apoptosis in mutiple myeloma. Blood 2001; 98 : 2183-92.

32. Berke N, Keklikoğlu N. Nöral krest kökenli hücrelerin ağiz ve çeneyüz oluşumuna katkilari ve neden olduklari anomaliler. JIUFD 2010; 44: 39-43.

33. Luo R, An M, Arduini BL, Henion PD. Specific pan-neural crest expression of zebrafish Crestin throughout embryonic development. Dev Dyn 2001; 220(2): 169-74. 